\title{
Synthesis, Thermal Stability, and Linear and Nonlinear Optical Properties of Hyperbranched Polyarylenes Containing Carbazole and/or Fluorene Moieties
}

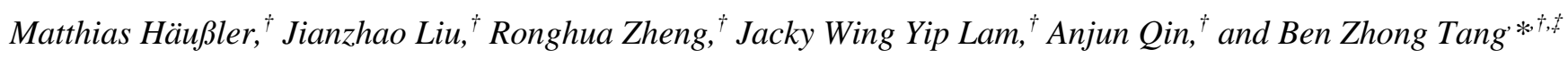

Department of Chemistry, The Hong Kong University of Science \& Technology, Clear Water Bay, Kowloon, Hong Kong, China, and Department of Polymer Science and Engineering, Zhejiang University, Hangzhou 310027, China

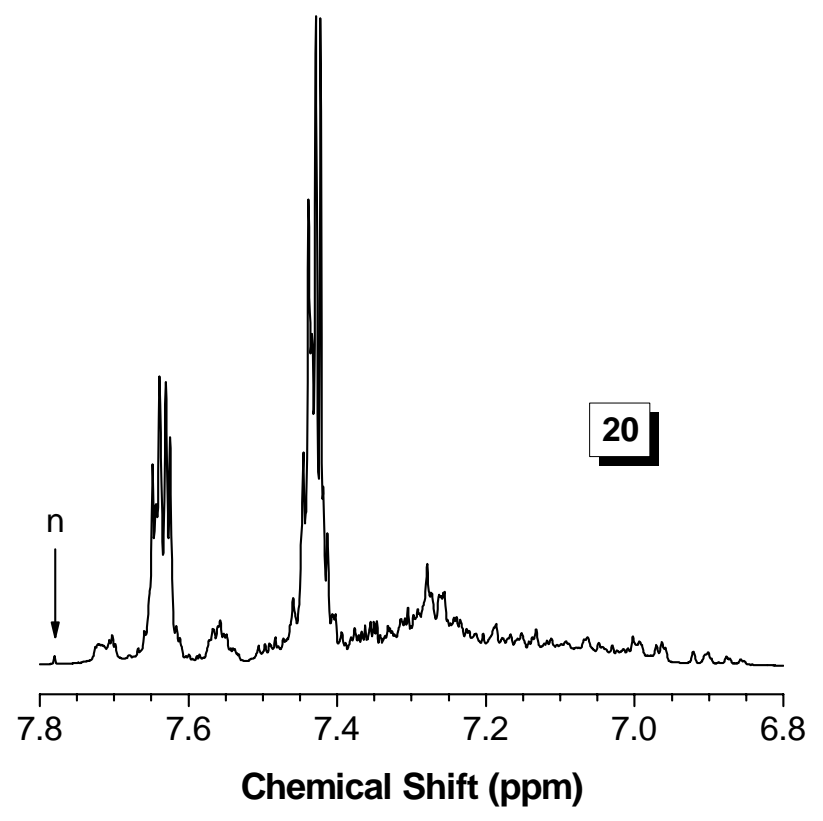

Figure S1. Aromatic proton resonance region in the ${ }^{1} \mathrm{H}$ NMR spectrum of the simulated substructures obtained from cocyclotrimerization of $\mathbf{1 7}$ with $\mathbf{4}$ in a molar feed ratio of 2:1.5. 